\title{
Evaluate the Sensory Parameters and Economics of Mixed Fruit Toffee
}

\author{
Bharat Kumar*, C.S. Pandey and N.R. Rangare \\ Department of Horticulture, Jawaharlal Nehru Krishi Vishwa Vidyalaya, Krishi Nagar \\ Adhartal, Jabalpur-482004, Madhya Pradesh
}

*Corresponding author:

\section{A B S T R A C T}

\begin{tabular}{|l|}
\hline Key w or d s \\
Mango, Papaya, \\
Blending, Toffee, \\
Economics \\
\hline Article Info \\
\hline Accepted: \\
26 July 2018 \\
Available Online: \\
10 August 2018 \\
\hline
\end{tabular}

The present investigation was carried out in the post-Harvest laboratory, Department of Horticulture, college of Agriculture, JNKVV Jabalpur (M.P) during the year 2014-2015. The Main objectives of the present investigation were to find out the best recipe with a suitable ratio of pulp and sugar for preparation of mixed fruit toffee. The mixed fruit toffee was prepared from mango and papaya pulp at different proportion viz. Comprised of 16 treatment combinations having 4 levels of mango and papaya pulp ratio (80:20, 60:40, 40:60 and 20:80) and 4 levels of sugar $(200 \mathrm{~g}, 300 \mathrm{~g}, 400 \mathrm{~g}, 500 \mathrm{~g})$, replicated thrice in Factorial Complete Randomized Design. Among the various treatments (recipe) treatment combination $\mathrm{C}_{2} \mathrm{~S}_{3}(60 \%$ mango $+40 \%$ papaya with $400 \mathrm{~g}$ sugar) was found best for organoleptic parameter based on taste, texture and overall acceptability. Whereas, $\mathrm{C}_{1} \mathrm{~S}_{3}$ $\left(80 \%\right.$ mango $+20 \%$ papaya with $400 \mathrm{~g}$ sugar) was found best in flavor and $\mathrm{C}_{4} \mathrm{~S}_{1}(20 \%$ mango $+80 \%$ papaya with $200 \mathrm{~g}$ sugar) best in colour and. The highest net return of Rs. 271.76 and $B: C$ ratio of 3.11 was obtained in the treatment combination $\mathrm{C}_{2} \mathrm{~S}_{3}$ due to maximum overall acceptability and higher sale rate.

\section{Introduction}

Fruits are very delicious to be used in fresh, processed and value added forms. Fruits are commercially important and nutritionally indispensable food commodity. Man has kept these commodities in his diet to provide variety, taste, interest, aesthetic appeal and to meet certain nutritional requirements.

Mango (Mangifera indica L.) is the most important and commercially cultivated fruit crop. Mango pulp is rich in the essential minerals, vitamins and other nutritive factors. It is an excellent source of vitamin A (4800
IU/100g) and vitamin C, vitamin B complex, iron, phosphorus etc. Besides, its attractive form and appearance, delicious taste and appetizing flavour, the ripe mango fruit, according to nutritional experts, is also highly invigorating, laxative and diuretic.

Some of the key components that contribute for the production and acceptance of high quality fresh mangoes by the consumer are flavour, volatiles, texture and chemical constituents (Tedjo et al., 2002; Mamiro et al., 2007). Due to shorter shelf life of the mango, it must be converted into various processed products. It is processed to make juices, jams, 
squashes, nectars, chutney, pickles, toffees, canned mango slices etc. (Hussain et al., 2005). Papaya (Carica papaya L.) regarded as the wonder fruit of the tropics and subtropics, belongs to the family Caricaceae and is originated in Mexico.

It is one of the most important fruits cultivated throughout the tropical and sub-tropical regions of the world (Anonymous, 2000).

It has got great importance due to its high nutritive value and production potentiality. In India, papaya occupies the area of 133 thousand ha having production of 5639 thousand MT with average productivity of 42.3 MT ha ${ }^{-1}$ fruit per year in 2013-14 (Anonymous, 2013-14).

Papaya is also a rich source of vitamin A (2020 IU/100g) and other vitamins like thiamine, riboflavin, nicotinic acid and ascorbic acid. Ripe papaya is also used in jam, jelly, marmalade and other products containing added sugar.

Other processed products include puree or wine, nectar (Matsuura et al., 2004), juice, frozen slices or chunks, mixed beverages, papaya powder, ice-cream, toffee, baby food, concentrated and candied items.

\section{Materials and Methods}

The fresh, uniform size, mature fruits of mango (cv. Neelum) and papaya (cv. Taiwan) were procured from the wholesale fruit market and sugar, vanaspati ghee, skim milk powder also were also purchased from local market used for experimentation.

The unripe, sorted diseased, damaged and off type fruits were discarded. The best quality/sorted fruits were picked up and used for the purpose of experimentation.

\section{Extraction of pulp}

The mature and ripe mango and papaya fruits were peeled using stainless steel knife and cut into small pieces and then the seed were discarded. $200 \mathrm{ml}$ water was added to each one $\mathrm{kg}$ of fruit pulp. Then heated up to 60 to $65{ }^{\circ} \mathrm{C}$ temperatures for 10 minutes and allowed to cool. The cooled pulp was passed through stainless steel sieve to obtain a pulp of papaya and seed and fiber free pulp of mango.

\section{Preparation of toffee}

The freshly prepared mango and papaya pulp were used for preparation of mixed fruit toffee, four combinations of fruit pulps are $\mathrm{C}_{1}(80 \%$ Mango $+20 \%$ Papaya), $\mathrm{C}_{2}(60 \%$ Mango + $40 \%$ Papaya), $\mathrm{C}_{3}$ (40\% Mango $+60 \%$ Papaya) and $\mathrm{C}_{4}(20 \%$ Mango $+80 \%$ Papaya).

As per recipe, the mixed fruit toffee, contains mixed fruit pulp $1.0 \mathrm{~kg}$, sugar $(200 \mathrm{~g}, 300 \mathrm{~g}$, $400 \mathrm{~g}$ and $500 \mathrm{~g}$ ) concentration, skim milk powder $150 \mathrm{~g}$, vanaspati ghee $125 \mathrm{~g}$ as par except milk powder, all other ingredients given in recipe are mixed very well and the mass is cooked up to $80^{\circ}$ Brix. Milk powder is dissolved in a small quantity of water and made thick paste and mixed in above masses and cooked again up to $82-84^{0}$ Brix. The cooked mass is transferred in stainless steel plate which is already smeared with butter and the product is spread into a thin sheet of 1 to 2 $\mathrm{cm}$ thickness. This is left to cool and set for two to three hours. Then the solid sheet is cut into cubes of different shapes with a stainless steel knife and wrapped in colour butter paper.

\section{Storage}

The prepared mixed fruit toffee was stored in dried place at ambient temperature which ranged from $18.20^{\circ} \mathrm{C}$ (minimum) to $31.90^{\circ} \mathrm{C}$ (maximum). 


\section{Flow sheet for the preparation of mixed fruit toffee}

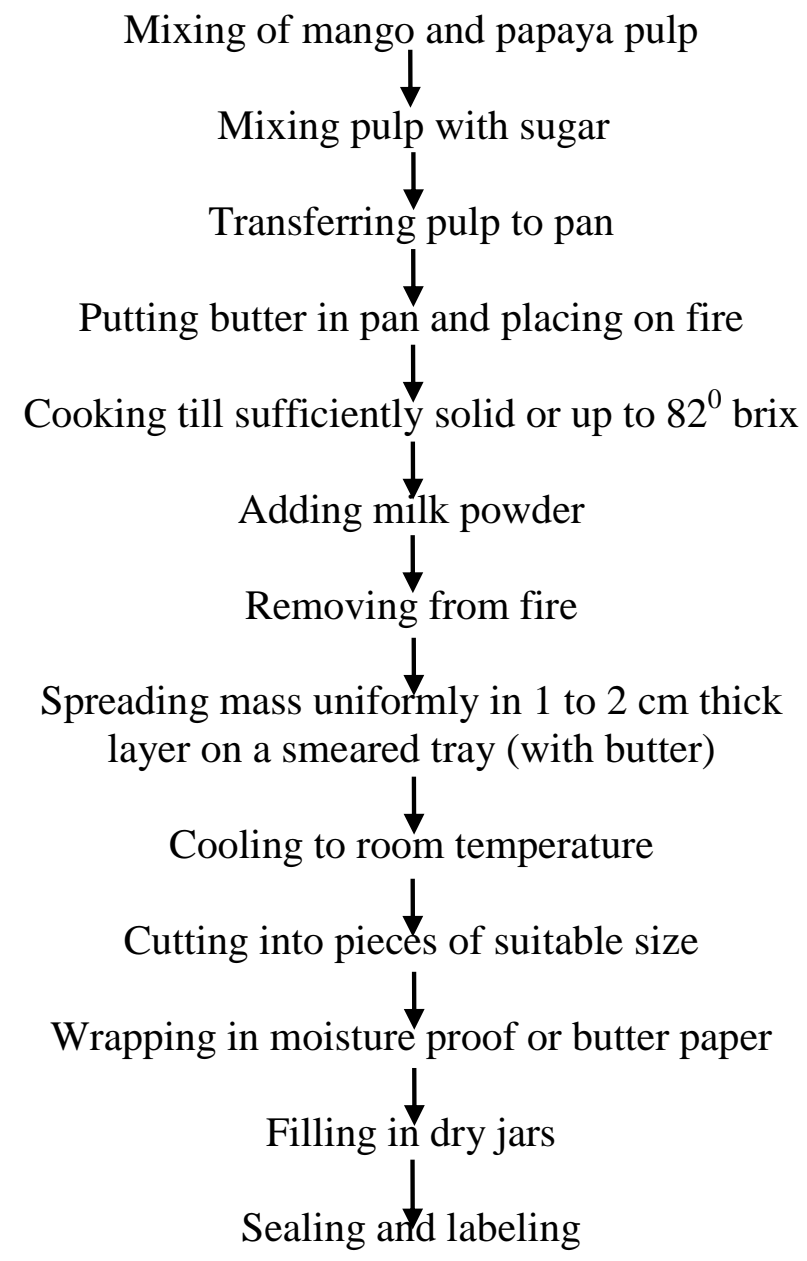

\section{Sensory evaluation of mixed fruit toffee}

A panel of 5 judges carried out the sensory evaluation for mixed fruit toffee. For the toffee different pulp concentration and recipes was evaluated for various sensory qualities attributes like colour, texture, flavour, taste and overall acceptability. The method was given by Amerine et al., (1965) was adopted with a 9 point hedonic scale.

\section{Evaluation of mixed fruit toffee:}

1. Colour

2. Texture

3. Flavour

4. Taste

5. Over all acceptability

\section{Economics of the treatment}

The economics of the treatments was estimated as per the actual cost of production and the market value of the product.

\section{Cost of production}

The cost on preparation of mixed fruit toffee (Mango and Papaya) was calculated by taking into Consideration the cost of all the inputs used and expenditure involved in the extraction of pulp from the fruits. The cost of production has been calculated for $1 \mathrm{~kg}$ of fruit pulp as per pulp ratio along with required different sugar quantity, labour charges, fuel 
and other expenses for the preparation of the mixed fruit toffee.

\section{Gross return (Rs.)}

The selling price for each treatment was worked out with the estimated market value of toffee as per quality and worked out for gross return.

\section{Benefit cost ratio}

It is the ratio of gross return (Rs) to the cost of product $(\mathrm{Rs} / \mathrm{kg})$. It is expressed in returns per rupee invested and presented for mixed fruit toffee.

Benefit Cost Ratio (B: C) $=$ Gross return (Rs) / Cost of product ( $\mathrm{Rs} / \mathrm{kg})$

\section{Results and Discussion}

The sensory characteristics of mixed fruit toffee were recorded and presented in various Tables. The quality of mixed fruit toffee depends upon its appearance (colour, flavour, taste, overall acceptability, etc.). Results of the organoleptic evaluation of mixed fruit toffee revealed that all the treatments were found acceptable during whole storage period.

\section{Colour}

The data presented in Table 1. clearly indicated that all treatments have slight differences in colour and colour rating value of mixed fruit toffee diminished gradually during storage from 0 to 120 days. Decrease of colour in stored mixed fruit toffee might be due to emphatic browning during storage. Browning of the toffee could have resulted from non enzymatic oxidation of vitamin $\mathrm{C}$ and enzymatic oxidation of polyphenols. Similar findings were obtained by Thakre and Jain (2013) in the blended nectar (50:50) of papaya and banana which was acceptable only for 15 days under ambient condition and up to 45 days under refrigerated condition.
According to them formation of brown pigment might be responsible for deterioration of colour of product. Similarly Sakhale et al., (2012)) observed that colour of whey based mango beverage decreased due to changes occurred during storage of beverage. Similar results were found by Singh et al., (2013) with jam prepared from three mango cultivars. This Different blend ratio of mango and papaya pulp and different concentration of sugar might be the reason of the difference in colour rating values. Highest colour rating value was observed with $20 \%$ mango $+80 \%$ papaya, perhaps due to dominant effect of papaya blending for colour appearance.

\section{Texture}

The highest value for texture was found in $\mathrm{C}_{2} \mathrm{~S}_{3}$ (60\% mango $+40 \%$ papaya with $400 \mathrm{~g}$ sugar) while minimum in $\mathrm{C}_{4} \mathrm{~S}_{1}(20 \%$ mango + $80 \%$ papaya with $200 \mathrm{~g}$ sugar). The results exhibited that higher proportion of mango pulp in comparison to papaya pulp was found better in improving the texture of mixed fruit toffee. In addition to it, the higher quantity of sugar up to $400 \mathrm{~g}$ gave better texture of toffee. As storage period increased, a very slight change in texture of mixed fruit toffee was observed. This might be due to absorption of moisture at the time of sensory evaluation and formation of brown pigment might be responsible for deterioration of appearance of product. Similar results were found i.e. decrease in texture by Singh et al., (2013) with jam prepared from three mango cultivars. Similar conclusions were drawn by Che man and Taufik (1995) with jackfruit leather and Aruna et al., (1999) during storage of papaya fruit bar (Table 2).

\section{Flavour}

The mean panelist score for flavour profile of mixed fruit toffee under storage indicated a decreasing trend. 
Int.J.Curr.Microbiol.App.Sci (2018) 7(8): $4321-4333$

Table.1 Effect of various recipes on colour of mixed fruit toffee during storage

\begin{tabular}{|c|c|c|c|c|c|c|c|c|c|c|c|c|c|c|c|c|c|c|c|c|c|c|c|c|c|}
\hline \multirow{2}{*}{$\begin{array}{c}\text { Concentration } \\
\text { of sugar }(\mathrm{g}) / \mathrm{kg} \\
\text { pulp } \\
\text { Factor B }\end{array}$} & \multicolumn{5}{|c|}{$\begin{array}{c}\text { '0' Day } \\
\text { Ratio of fruit pulp } \\
\text { (Mango \% + Papaya \%) } \\
\text { Factor A }\end{array}$} & \multicolumn{5}{|c|}{$\begin{array}{c}\text { '30' Days } \\
\text { Ratio of fruit pulp } \\
(\text { Mango \% + Papaya \%) } \\
\text { Factor A }\end{array}$} & \multicolumn{5}{|c|}{$\begin{array}{c}\text { '60' Days } \\
\text { Ratio of fruit pulp } \\
\text { (Mango \% + Papaya \%) } \\
\text { Factor A }\end{array}$} & \multicolumn{5}{|c|}{$\begin{array}{c}\text { '90' Days } \\
\text { Ratio of fruit pulp } \\
\text { (Mango \%+Papaya \%) } \\
\text { Factor A }\end{array}$} & \multicolumn{5}{|c|}{$\begin{array}{c}\text { ' } 120 \text { ' Days } \\
\text { Ratio of fruit pulp } \\
(\text { Mango \% + Papaya \%) } \\
\text { Factor A }\end{array}$} \\
\hline & $\begin{array}{c}C_{1} \\
80: 20\end{array}$ & $\begin{array}{c}C_{2} \\
60: 40\end{array}$ & $\begin{array}{c}C_{3} \\
40: 60\end{array}$ & $\begin{array}{c}C_{4} \\
20: 80\end{array}$ & Mean & $\begin{array}{c}\mathrm{C}_{1} \\
\mathbf{8 0 : 2 0}\end{array}$ & $\begin{array}{c}C_{2} \\
60: 40\end{array}$ & $\begin{array}{c}C_{3} \\
40: 60\end{array}$ & $\begin{array}{c}C_{4} \\
20: 80\end{array}$ & Mean & $\begin{array}{c}C_{1} \\
80: 20\end{array}$ & $\begin{array}{c}C_{2} \\
60: 40\end{array}$ & $\begin{array}{c}C_{3} \\
40: 60\end{array}$ & $\begin{array}{c}\mathrm{C}_{4} \\
20: 80\end{array}$ & Mean & $\begin{array}{c}C_{1} \\
80: 20\end{array}$ & $\begin{array}{c}C_{2} \\
60: 40\end{array}$ & $\begin{array}{c}C_{3} \\
40: 60\end{array}$ & $\begin{array}{c}C_{4} \\
20: 80\end{array}$ & Mean & $\begin{array}{c}C_{1} \\
80: 20\end{array}$ & $\begin{array}{c}C_{2} \\
60: 40\end{array}$ & $\begin{array}{c}C_{3} \\
40: 60\end{array}$ & $\begin{array}{c}C_{4} \\
20: 80\end{array}$ & Mean \\
\hline$S_{1}(200 g)$ & 7.80 & 7.40 & 7.00 & 9.00 & 7.80 & 7.60 & 7.20 & 7.00 & 8.80 & 7.65 & 7.60 & 7.20 & 6.60 & 8.40 & 7.45 & 7.20 & 7.00 & 6.60 & 8.40 & 7.30 & 7.00 & 6.80 & 6.40 & 8.20 & 7.10 \\
\hline$S_{2}(300 g)$ & 7.80 & 7.40 & 7.20 & 8.80 & 7.80 & 7.80 & 7.20 & 7.00 & 8.60 & 7.65 & 7.60 & 7.20 & 6.80 & 8.40 & 7.50 & 7.20 & 7.00 & 6.80 & 8.20 & 7.30 & 7.00 & 6.80 & 6.40 & 8.00 & 7.05 \\
\hline$S_{3}(400 g)$ & 8.00 & 7.40 & 7.20 & 8.80 & 7.85 & 7.80 & 7.20 & 7.00 & 6.60 & 7.15 & 7.60 & 7.20 & 7.00 & 8.40 & 7.55 & 7.40 & 7.00 & 6.80 & 8.00 & 7.30 & 7.20 & 6.80 & 6.40 & 8.00 & 7.10 \\
\hline $\mathrm{S}_{4}(500 \mathrm{~g})$ & 8.20 & 7.60 & 7.80 & 8.60 & 8.05 & 8.00 & 7.60 & 7.60 & 8.20 & 7.85 & 7.80 & 7.40 & 7.60 & 8.00 & 7.70 & 7.60 & 7.20 & 7.20 & 8.00 & 7.50 & 7.40 & 7.00 & 7.00 & 7.80 & 7.30 \\
\hline Mean & 7.95 & 7.45 & 7.30 & 8.80 & & 7.80 & 7.30 & 7.15 & 8.05 & & 7.65 & 7.25 & 7.00 & 8.30 & & 7.35 & 7.05 & 6.85 & 8.15 & & 7.15 & 6.85 & 6.55 & 8.00 & \\
\hline Factor & A & B & $\mathrm{AB}$ & & & A & B & $\mathrm{AB}$ & & & A & B & $\mathrm{AB}$ & & & A & B & $\mathrm{AB}$ & & & A & B & $\mathrm{AB}$ & & \\
\hline SEm \pm & 0.066 & 0.066 & 0.133 & & & 0.062 & 0.062 & 0.124 & & & 0.060 & 0.060 & 0.120 & & & 0.058 & 0.058 & 0.116 & & & 0.059 & 0.059 & 0.118 & & \\
\hline CD at $5 \%$ level & 0.192 & 0.192 & 0.384 & & & 0.179 & 0.179 & 0.358 & & & 0.173 & 0.173 & 0.346 & & & 0.168 & 0.168 & 0.335 & & & 0.170 & 0.170 & 0.341 & & \\
\hline
\end{tabular}


Int.J.Curr.Microbiol.App.Sci (2018) 7(8): 4321-4333

Table.2 Effect of various recipes on texture of mixed fruit toffee during storage

\begin{tabular}{|c|c|c|c|c|c|c|c|c|c|c|c|c|c|c|c|c|c|c|c|c|c|c|c|c|c|}
\hline \multirow{2}{*}{$\begin{array}{c}\text { Concentration of } \\
\text { sugar }(\mathrm{g}) / \mathrm{kg} \text { pulp } \\
\text { Factor B }\end{array}$} & \multicolumn{5}{|c|}{$\begin{array}{c}\text { '0' Day } \\
\text { Ratio of fruit pulp } \\
\text { (Mango \% + Papaya \%) } \\
\text { Factor A }\end{array}$} & \multicolumn{5}{|c|}{$\begin{array}{c}\text { '30' Days } \\
\text { Ratio of fruit pulp } \\
(\text { Mango \% + Papaya \%) } \\
\text { Factor A }\end{array}$} & \multicolumn{5}{|c|}{$\begin{array}{c}\text { '60' Days } \\
\text { Ratio of fruit pulp } \\
\text { (Mango \% + Papaya \%) } \\
\text { Factor A }\end{array}$} & \multicolumn{5}{|c|}{$\begin{array}{c}\text { '90' Days } \\
\text { Ratio of fruit pulp } \\
\text { (Mango \%+Papaya \%) } \\
\text { Factor A }\end{array}$} & \multicolumn{5}{|c|}{$\begin{array}{c}\text { '120' Days } \\
\text { Ratio of fruit pulp } \\
(\text { Mango \% + Papaya \%) } \\
\text { Factor A }\end{array}$} \\
\hline & $\begin{array}{c}C_{1} \\
80: 20\end{array}$ & $\begin{array}{c}C_{2} \\
60: 40\end{array}$ & $\begin{array}{c}C_{3} \\
40: 60\end{array}$ & $\begin{array}{c}\mathrm{C}_{4} \\
20: 80\end{array}$ & Mean & $\begin{array}{c}C_{1} \\
80: 20\end{array}$ & $\begin{array}{c}\mathrm{C}_{2} \\
60: 40\end{array}$ & $\begin{array}{c}C_{3} \\
40: 60\end{array}$ & $\begin{array}{c}C_{4} \\
20: 80\end{array}$ & Mean & $\begin{array}{c}\mathrm{C}_{1} \\
80: 20\end{array}$ & $\begin{array}{c}C_{2} \\
60: 40\end{array}$ & $\begin{array}{c}C_{3} \\
40: 60\end{array}$ & $\begin{array}{c}C_{4} \\
20: 80\end{array}$ & Mean & $\begin{array}{c}C_{1} \\
80: 20\end{array}$ & $\begin{array}{c}C_{2} \\
60: 40\end{array}$ & $\mid \begin{array}{c}C_{3} \\
40: 60\end{array}$ & $\begin{array}{c}C_{4} \\
20: 80\end{array}$ & Mean & $\begin{array}{c}\mathrm{C}_{1} \\
80: 20\end{array}$ & $\begin{array}{c}C_{2} \\
60: 40\end{array}$ & $\begin{array}{c}C_{3} \\
40: 60\end{array}$ & $\begin{array}{c}\mathrm{C}_{4} \\
20: 80\end{array}$ & Mean \\
\hline$S_{1}(200 g)$ & 7.60 & 7.80 & 7.60 & 7.00 & 7.50 & 7.20 & 7.40 & 7.40 & 6.80 & 7.20 & 7.00 & 7.20 & 7.20 & 6.60 & 7.00 & 6.80 & 7.00 & 6.80 & 6.20 & 6.70 & 6.60 & 6.80 & 6.60 & 5.80 & 6.45 \\
\hline$S_{2}(300 g)$ & 7.60 & 7.80 & 8.00 & 7.20 & 7.70 & 7.20 & 7.80 & 7.60 & 6.80 & 7.35 & 7.00 & 7.40 & 7.20 & 6.80 & 7.10 & 6.80 & 7.40 & 7.20 & 6.40 & 6.95 & 6.40 & 7.20 & 7.00 & 6.20 & 6.70 \\
\hline$S_{3}(400 g)$ & 8.00 & 8.20 & 8.00 & 7.20 & 7.85 & 7.80 & 8.20 & 7.60 & 7.00 & 7.65 & 7.40 & 7.80 & 7.20 & 6.80 & 7.30 & 7.20 & 7.80 & 7.00 & 6.40 & 7.10 & 7.20 & 7.60 & 6.80 & 6.20 & 6.95 \\
\hline $\mathrm{S}_{4}(\mathbf{5 0 0 g})$ & 8.00 & 7.80 & 7.80 & 7.60 & 7.85 & 7.80 & 7.40 & 7.60 & 7.20 & 7.50 & 7.40 & 7.20 & 7.20 & 7.00 & 7.20 & 7.20 & 7.00 & 7.00 & 6.80 & 7.00 & 7.20 & 6.60 & 6.80 & 6.40 & 6.75 \\
\hline Mean & 7.80 & 7.95 & 7.85 & 7.25 & & 7.50 & 7.70 & 7.55 & 6.95 & & 7.20 & 7.40 & 7.20 & 6.80 & & 7.00 & 7.30 & 7.00 & 6.45 & & 6.85 & 7.05 & 6.80 & 6.15 & \\
\hline Factor & A & B & $\mathrm{AB}$ & & & A & B & $\mathrm{AB}$ & & & A & B & $\mathrm{AB}$ & & & A & B & $\mathrm{AB}$ & & & A & B & $\mathrm{AB}$ & & \\
\hline SEm \pm & 0.053 & 0.053 & 0.105 & & & 0.054 & 0.054 & 0.107 & & & 0.059 & 0.059 & 0.119 & & & 0.066 & 0.066 & 0.132 & & & 0.060 & 0.060 & 0.121 & & \\
\hline CD at $5 \%$ level & 0.152 & 0.152 & 0.304 & & & 0.155 & 0.155 & 0.311 & & & 0.172 & 0.172 & 0.172 & & & 0.191 & 0.191 & 0.381 & & & 0.174 & 0.174 & 0.349 & & \\
\hline
\end{tabular}


Int.J.Curr.Microbiol.App.Sci (2018) 7(8): 4321-4333

Table.3 Effect of various recipes on flavour of mixed fruit toffee during storage

\begin{tabular}{|c|c|c|c|c|c|c|c|c|c|c|c|c|c|c|c|c|c|c|c|c|c|c|c|c|c|}
\hline \multirow[t]{2}{*}{$\begin{array}{c}\begin{array}{c}\text { Concentration } \\
\text { of sugar }(\mathrm{g}) / \mathrm{kg}\end{array} \\
\text { pulp } \\
\text { Factor B }\end{array}$} & \multicolumn{5}{|c|}{$\begin{array}{c}\text { '0' Day } \\
\text { Ratio of fruit pulp } \\
\text { (Mango \% + Papaya \%) } \\
\text { Factor A }\end{array}$} & \multicolumn{5}{|c|}{$\begin{array}{c}\text { '30' Days } \\
\text { Ratio of fruit pulp } \\
\text { (Mango \% + Papaya \%) } \\
\text { Factor A }\end{array}$} & \multicolumn{5}{|c|}{$\begin{array}{c}\text { '60' Days } \\
\text { Ratio of fruit pulp } \\
\text { (Mango \% + Papaya \%) } \\
\text { Factor A }\end{array}$} & \multicolumn{5}{|c|}{$\begin{array}{c}\text { ‘90' Days } \\
\text { Ratio of fruit pulp } \\
\text { (Mango \%+Papaya \%) } \\
\text { Factor A }\end{array}$} & \multicolumn{5}{|c|}{$\begin{array}{c}\text { ' } 120 \text { ' Days } \\
\text { Ratio of fruit pulp } \\
(\text { Mango \% + Papaya \%) } \\
\text { Factor A }\end{array}$} \\
\hline & $\begin{array}{c}C_{1} \\
80: 20\end{array}$ & $\begin{array}{c}C_{2} \\
60: 40\end{array}$ & $\begin{array}{c}C_{3} \\
40: 60\end{array}$ & $\begin{array}{c}\mathrm{C}_{4} \\
\text { 20:80 }\end{array}$ & Mean & $\begin{array}{c}C_{1} \\
80: 20\end{array}$ & $\begin{array}{c}C_{2} \\
60: 40\end{array}$ & $\begin{array}{c}C_{3} \\
40: 60\end{array}$ & $\begin{array}{c}\mathrm{C}_{4} \\
\text { 20:80 }\end{array}$ & Mean & $\begin{array}{c}\mathrm{C}_{1} \\
80: 20\end{array}$ & $\begin{array}{c}C_{2} \\
60: 40\end{array}$ & $\begin{array}{c}C_{3} \\
40: 60\end{array}$ & $\begin{array}{c}\mathrm{C}_{4} \\
20: 80\end{array}$ & Mean & $\begin{array}{c}\mathrm{C}_{1} \\
80: 20\end{array}$ & $\begin{array}{c}C_{2} \\
60: 40\end{array}$ & $\begin{array}{c}C_{3} \\
40: 60\end{array}$ & $\begin{array}{c}\mathrm{C}_{4} \\
20: 80\end{array}$ & Mean & $\begin{array}{c}C_{1} \\
80: 20\end{array}$ & $\begin{array}{c}C_{2} \\
60: 40\end{array}$ & $\begin{array}{c}C_{3} \\
40: 60\end{array}$ & $\begin{array}{c}\mathrm{C}_{4} \\
20: 80\end{array}$ & Mean \\
\hline$S_{1}(200 g)$ & 8.40 & 7.80 & 7.60 & 7.00 & 7.70 & 8.20 & 7.80 & 7.20 & 6.80 & 7.50 & 8.00 & 7.60 & 7.00 & 6.60 & 7.30 & 7.80 & 7.20 & 6.60 & 6.40 & 7.00 & 7.80 & 7.00 & 6.40 & 6.00 & 6.80 \\
\hline$S_{2}(300 g)$ & 8.40 & 7.80 & 7.40 & 7.20 & 7.70 & 8.40 & 7.80 & 7.00 & 7.00 & 7.55 & 8.20 & 7.60 & 6.80 & 6.60 & 7.30 & 8.00 & 7.20 & 6.60 & 6.40 & 7.05 & 8.00 & 7.20 & 6.40 & 6.20 & 6.95 \\
\hline$S_{3}(400 g)$ & 8.60 & 8.00 & 7.60 & 7.40 & 7.90 & 8.60 & 7.80 & 7.20 & 7.00 & 7.65 & 8.40 & 7.80 & 7.00 & 6.60 & 7.45 & 8.40 & 7.60 & 6.60 & 6.40 & 7.25 & 8.20 & 7.20 & 6.40 & 6.20 & 7.00 \\
\hline$S_{4}(500 g)$ & 8.40 & 8.20 & 7.60 & 7.40 & 7.90 & 8.40 & 8.20 & 7.20 & 7.00 & 7.70 & 8.40 & 7.80 & 7.00 & 6.60 & 7.45 & 8.20 & 7.80 & 6.80 & 6.40 & 7.30 & 8.00 & 7.40 & 6.60 & 6.20 & 7.05 \\
\hline Mean & 8.45 & 7.95 & 7.55 & 7.25 & & 8.40 & 7.90 & 7.15 & 6.95 & & 8.25 & 7.70 & 6.95 & 6.60 & & 8.10 & 7.45 & 6.65 & 6.40 & & 8.00 & 7.20 & 6.45 & 6.15 & \\
\hline Factor & A & B & $\mathrm{AB}$ & & & A & B & $\mathrm{AB}$ & & & A & B & $\mathrm{AB}$ & & & A & B & $\mathrm{AB}$ & & & A & B & $\mathrm{AB}$ & & \\
\hline SEm \pm & 0.050 & 0.050 & 0.100 & & & 0.053 & 0.053 & 0.105 & & & 0.050 & 0.050 & 0.100 & & & 0.055 & 0.055 & 0.110 & & & 0.055 & 0.055 & 0.110 & & \\
\hline CD at $5 \%$ level & 0.145 & 0.145 & NS & & & 0.152 & 0.152 & NS & & & 0.145 & 0.145 & NS & & & 0.160 & 0.160 & NS & & & 0.159 & 0.159 & NS & & \\
\hline
\end{tabular}


Int.J.Curr.Microbiol.App.Sci (2018) 7(8): 4321-4333

Table.4 Effect of various recipes on taste of mixed fruit toffee during storage

\begin{tabular}{|c|c|c|c|c|c|c|c|c|c|c|c|c|c|c|c|c|c|c|c|c|c|c|c|c|c|}
\hline \multirow{2}{*}{$\begin{array}{c}\text { Concentration } \\
\text { of sugar }(\mathrm{g}) / \mathrm{kg} \\
\text { pulp } \\
\text { Factor B }\end{array}$} & \multicolumn{5}{|c|}{$\begin{array}{c}\text { '0' Day } \\
\text { Ratio of fruit pulp } \\
\text { (Mango \% + Papaya \%) } \\
\text { Factor A }\end{array}$} & \multicolumn{5}{|c|}{$\begin{array}{c}\text { '30' Days } \\
\text { Ratio of fruit pulp } \\
\text { (Mango \% + Papaya \%) } \\
\text { Factor A }\end{array}$} & \multicolumn{5}{|c|}{$\begin{array}{c}\text { '60' Days } \\
\text { Ratio of fruit pulp } \\
\text { (Mango \% + Papaya \%) } \\
\text { Factor A }\end{array}$} & \multicolumn{5}{|c|}{$\begin{array}{c}\text { '90' Days } \\
\text { Ratio of fruit pulp } \\
\text { (Mango \%+Papaya \%) } \\
\text { Factor A }\end{array}$} & \multicolumn{5}{|c|}{$\begin{array}{c}\text { ' } 120 \text { ' Days } \\
\text { Ratio of fruit pulp } \\
\text { (Mango \% + Papaya \%) } \\
\text { Factor A }\end{array}$} \\
\hline & $\begin{array}{c}\mathrm{C}_{1} \\
80: 20\end{array}$ & $\begin{array}{c}C_{2} \\
60: 40\end{array}$ & $\begin{array}{c}C_{3} \\
40: 60\end{array}$ & $\begin{array}{c}C_{4} \\
20: 80\end{array}$ & Mean & $\begin{array}{c}C_{1} \\
80: 20\end{array}$ & $\begin{array}{c}\mathrm{C}_{2} \\
60: 40\end{array}$ & $\begin{array}{c}C_{3} \\
40: 60\end{array}$ & $\begin{array}{c}\mathrm{C}_{4} \\
20: 80\end{array}$ & Mean & $\begin{array}{c}C_{1} \\
80: 20\end{array}$ & $\begin{array}{c}C_{2} \\
60: 40\end{array}$ & $\begin{array}{c}C_{3} \\
40: 60\end{array}$ & $\begin{array}{c}\mathrm{C}_{4} \\
20: 80\end{array}$ & Mean & $\begin{array}{c}C_{1} \\
80: 20\end{array}$ & $\begin{array}{c}C_{2} \\
60: 40\end{array}$ & $\begin{array}{c}C_{3} \\
40: 60\end{array}$ & $\begin{array}{c}C_{4} \\
20: 80\end{array}$ & Mean & $\begin{array}{c}\mathrm{C}_{1} \\
80: 20\end{array}$ & $\begin{array}{c}C_{2} \\
60: 40\end{array}$ & $\begin{array}{c}C_{3} \\
40: 60\end{array}$ & $\begin{array}{c}\mathrm{C}_{4} \\
20: 80\end{array}$ & Mean \\
\hline $\mathrm{S}_{1}(200 \mathrm{~g})$ & 7.60 & 8.00 & 7.80 & 7.00 & 7.60 & 7.40 & 8.00 & 7.60 & 6.60 & 7.40 & 7.20 & 7.60 & 7.40 & 6.60 & 7.20 & 7.00 & 7.40 & 7.00 & 6.40 & 6.95 & 7.00 & 7.20 & 7.00 & 6.20 & 6.85 \\
\hline$S_{2}(300 g)$ & 7.40 & 8.80 & 8.40 & 7.20 & 7.95 & 7.40 & 8.80 & 8.20 & 7.20 & 7.90 & 7.20 & 8.40 & 8.00 & 7.00 & 7.65 & 7.00 & 8.40 & 7.60 & 6.80 & 7.45 & 6.80 & 8.00 & 7.60 & 6.60 & 7.25 \\
\hline$S_{3}(400 g)$ & 8.60 & 9.00 & 8.40 & 7.40 & 8.35 & 8.40 & 8.80 & 8.20 & 7.20 & 8.15 & 8.40 & 8.60 & 8.00 & 7.00 & 8.00 & 8.20 & 8.40 & 7.60 & 6.80 & 7.75 & 7.80 & 8.20 & 7.40 & 6.60 & 7.50 \\
\hline Mean & 8.05 & 8.40 & 8.20 & 7.25 & & 7.90 & 8.30 & 8.00 & 7.05 & & 7.70 & 8.00 & 7.75 & 6.90 & & 7.55 & 7.85 & 7.40 & 6.70 & & 7.30 & 7.60 & 7.35 & 6.55 & \\
\hline Factor & A & B & $\mathrm{AB}$ & & & A & B & $\mathrm{AB}$ & & & A & B & $\mathrm{AB}$ & & & A & B & $\mathrm{AB}$ & & & A & B & $\mathrm{AB}$ & & \\
\hline SEm \pm & 0.161 & 0.161 & 0.321 & & & 0.158 & 0.158 & 0.317 & & & 0.155 & 0.155 & 0.310 & & & 0.152 & 0.152 & 0.304 & & & 0.150 & 0.150 & 0.299 & & \\
\hline CD at $5 \%$ level & 0.465 & 0.465 & NS & & & 0.458 & 0.458 & NS & & & 0.449 & 0.449 & NS & & & 0.440 & 0.440 & NS & & & 0.433 & 0.433 & NS & & \\
\hline
\end{tabular}


Table.5 Effect of various recipes on overall acceptability of mixed fruit toffee during storage

\begin{tabular}{|c|c|c|c|c|c|c|c|c|c|c|c|c|c|c|c|c|c|c|c|c|c|c|c|c|c|}
\hline \multirow{2}{*}{\begin{tabular}{|c} 
Concentration \\
of sugar \\
$(\mathrm{g}) / \mathrm{kg}$ pulp \\
Factor B
\end{tabular}} & \multicolumn{5}{|c|}{$\begin{array}{c}\text { '0' Day } \\
\text { Ratio of fruit pulp } \\
\text { (Mango \% + Papaya \%) } \\
\text { Factor A }\end{array}$} & \multicolumn{5}{|c|}{$\begin{array}{c}\text { '30' Days } \\
\text { Ratio of fruit pulp } \\
\text { (Mango \% + Papaya \%) } \\
\text { Factor A }\end{array}$} & \multicolumn{5}{|c|}{$\begin{array}{c}\text { ‘60’ Days } \\
\text { Ratio of fruit pulp } \\
\text { (Mango \% + Papaya \%) } \\
\text { Factor A }\end{array}$} & \multicolumn{5}{|c|}{$\begin{array}{c}\text { ‘90' Days } \\
\text { Ratio of fruit pulp } \\
\text { (Mango \%+Papaya \%) } \\
\text { Factor A }\end{array}$} & \multicolumn{5}{|c|}{$\begin{array}{c}\text { '120' Days } \\
\text { Ratio of fruit pulp } \\
\text { (Mango \% + Papaya \%) } \\
\text { Factor A }\end{array}$} \\
\hline & $\begin{array}{c}\mathrm{C}_{1} \\
80: 20\end{array}$ & $\begin{array}{c}C_{2} \\
60: 40\end{array}$ & $\begin{array}{c}C_{3} \\
40: 60\end{array}$ & $\begin{array}{c}C_{4} \\
20: 80\end{array}$ & Mean & $\begin{array}{c}C_{1} \\
80: 20\end{array}$ & $\begin{array}{c}C_{2} \\
60: 40\end{array}$ & $\begin{array}{c}C_{3} \\
40: 60\end{array}$ & $\begin{array}{c}C_{4} \\
20: 80\end{array}$ & Mean & $\begin{array}{c}C_{1} \\
80: 20\end{array}$ & $\begin{array}{c}C_{2} \\
60: 40\end{array}$ & $\begin{array}{c}C_{3} \\
40: 60\end{array}$ & $\begin{array}{c}C_{4} \\
20: 80\end{array}$ & Mean & $\begin{array}{c}C_{1} \\
80: 20\end{array}$ & $\begin{array}{c}C_{2} \\
60: 40\end{array}$ & $\begin{array}{c}C_{3} \\
40: 60\end{array}$ & $\begin{array}{c}C_{4} \\
20: 80\end{array}$ & Mean & $\begin{array}{c}C_{1} \\
80: 20\end{array}$ & $\begin{array}{c}C_{2} \\
60: 40\end{array}$ & $\begin{array}{c}C_{3} \\
40: 60\end{array}$ & $\mid \begin{array}{c}C_{4} \\
20: 80\end{array}$ & Mean \\
\hline$S_{1}(200 g)$ & 7.40 & 7.60 & 7.40 & 6.80 & 7.30 & 7.00 & 7.40 & 7.00 & 6.60 & 7.00 & 6.80 & 7.20 & 7.00 & 6.20 & 6.80 & 6.60 & 7.00 & 6.60 & 6.00 & 6.55 & 6.20 & 6.80 & 6.40 & 5.80 & 6.30 \\
\hline$S_{2}(300 g)$ & 7.40 & 7.80 & 7.60 & 7.00 & 7.45 & 7.00 & 7.80 & 7.40 & 6.80 & 7.25 & 6.80 & 7.60 & 7.40 & 6.60 & 7.10 & 6.60 & 7.40 & 7.20 & 6.40 & 6.90 & 6.20 & 7.20 & 7.20 & 6.00 & 6.65 \\
\hline$S_{3}(400 g)$ & 7.80 & 8.00 & 7.60 & 7.00 & 7.60 & 7.60 & 8.00 & 7.40 & 6.80 & 7.45 & 7.60 & 7.80 & 7.20 & 6.60 & 7.30 & 7.20 & 7.60 & 7.20 & 6.60 & 7.15 & 7.20 & 7.60 & 7.20 & 6.20 & 7.05 \\
\hline$S_{4}(500 g)$ & 7.80 & 7.40 & 7.60 & 7.20 & 7.50 & 7.60 & 7.20 & 7.40 & 7.00 & 7.30 & 7.60 & 7.00 & 7.20 & 6.80 & 7.15 & 7.20 & 6.80 & 7.00 & 6.60 & 6.90 & 7.20 & 6.80 & 6.80 & 6.20 & 6.75 \\
\hline Mean & 7.60 & 7.70 & 7.55 & 7.00 & & 7.30 & 7.60 & 7.30 & 6.80 & & 7.20 & 7.40 & 7.20 & 6.55 & & 6.90 & 7.20 & 7.00 & 6.40 & & 6.70 & 7.10 & 6.90 & 6.05 & \\
\hline Factor & A & B & $\mathrm{AB}$ & & & A & B & $\mathrm{AB}$ & & & A & B & $\mathrm{AB}$ & & & A & B & $\mathrm{AB}$ & & & A & B & $\mathrm{AB}$ & & \\
\hline $\mathrm{SEm} \pm$ & 0.053 & 0.053 & 0.105 & & & 0.053 & 0.053 & 0.105 & & & 0.050 & 0.050 & 0.100 & & & 0.050 & 0.050 & 0.100 & & & 0.062 & 0.062 & 0.124 & & \\
\hline $\begin{array}{c}\text { CD at } \\
5 \% \text { level }\end{array}$ & 0.152 & 0.152 & 0.304 & & & 0.152 & 0.152 & 0.304 & & & 0.145 & 0.145 & 0.289 & & & 0.145 & 0.145 & 0.289 & & & 0.179 & 0.179 & 0.358 & & \\
\hline
\end{tabular}


Table.6 Economics of various treatments for mixed fruit toffee

\begin{tabular}{|c|c|c|c|c|c|c|c|c|c|c|}
\hline Treatments & $\begin{array}{c}\text { Cost of } \\
\text { fruit } \\
\text { pulp } \\
\text { (Rs.) }\end{array}$ & $\begin{array}{c}\text { Cost of } \\
\text { sugar } \\
\text { (Rs.) }\end{array}$ & $\begin{array}{c}\text { Cost of } \\
\text { vanspati } \\
\text { ghee +milk } \\
\text { powder }\end{array}$ & $\begin{array}{c}\text { Processing } \\
\text { cost } \\
\text { (Rs.) }\end{array}$ & $\begin{array}{l}\text { Total cost } \\
\quad \text { (Rs.) }\end{array}$ & $\begin{array}{c}\text { Gross } \\
\text { return } \\
\text { (Rs.) }\end{array}$ & $\begin{array}{c}\text { Net } \\
\text { return } \\
(\mathbf{R s .})\end{array}$ & $\begin{array}{l}\mathrm{B}: \mathrm{C} \\
\text { ratio }\end{array}$ & $\begin{array}{l}\text { No. Of } \\
\text { Toffee }\end{array}$ & $\begin{array}{c}\text { Sale } \\
\operatorname{rate}(\mathbf{R s .} .) *\end{array}$ \\
\hline $\mathbf{T}_{1}$ & 39.87 & 6 & 63.13 & 15 & 124.00 & 205 & 81.00 & 1.65 & 205 & 1.00 \\
\hline $\mathbf{T}_{2}$ & 39.87 & 9 & 63.13 & 15 & 127.00 & 295 & 168.00 & 2.32 & 236 & 1.25 \\
\hline $\mathbf{T}_{3}$ & 39.87 & 12 & 63.13 & 15 & 130.00 & 341.25 & 211.25 & 2.61 & 273 & 1.25 \\
\hline $\mathbf{T}_{4}$ & 39.87 & 15 & 63.13 & 15 & 133.00 & 348.75 & 215.75 & 2.62 & 279 & 1.25 \\
\hline $\mathbf{T}_{5}$ & 38.61 & 6 & 63.13 & 15 & 122.74 & 251.25 & 128.51 & 2.05 & 201 & 1.25 \\
\hline $\mathbf{T}_{6}$ & 38.61 & 9 & 63.13 & 15 & 125.74 & 319.50 & 193.76 & 2.54 & 213 & 1.50 \\
\hline $\mathbf{T}_{7}$ & 38.61 & 12 & 63.13 & 15 & 128.74 & 400.50 & 271.76 & 3.11 & 267 & 1.50 \\
\hline $\mathbf{T}_{8}$ & 38.61 & 15 & 63.13 & 15 & 131.74 & 335 & 203.26 & 2.54 & 268 & 1.25 \\
\hline $\mathbf{T}_{9}$ & 37.41 & 6 & 63.13 & 15 & 121.54 & 195 & 73.46 & 1.60 & 195 & 1.00 \\
\hline $\mathbf{T}_{\mathbf{1 0}}$ & 37.41 & 9 & 63.13 & 15 & 124.54 & 281.25 & 156.71 & 2.26 & 225 & 1.25 \\
\hline $\mathbf{T}_{11}$ & 37.41 & 12 & 63.13 & 15 & 127.54 & 301.25 & 173.71 & 2.36 & 241 & 1.25 \\
\hline $\mathbf{T}_{12}$ & 37.41 & 15 & 63.13 & 15 & 130.54 & 263 & 132.46 & 2.01 & 263 & 1.00 \\
\hline $\mathbf{T}_{13}$ & 36.18 & 6 & 63.13 & 15 & 120.31 & 183 & 62.69 & 1.52 & 183 & 1.00 \\
\hline $\mathbf{T}_{14}$ & 36.18 & 9 & 63.13 & 15 & 123.31 & 189 & 65.69 & 1.53 & 189 & 1.00 \\
\hline $\mathbf{T}_{15}$ & 36.18 & 12 & 63.13 & 15 & 126.31 & 212 & 85.69 & 1.68 & 212 & 1.00 \\
\hline $\mathbf{T}_{16}$ & 36.18 & 15 & 63.13 & 15 & 129.31 & 267 & 137.69 & 2.06 & 267 & 1.00 \\
\hline
\end{tabular}


It was also clear from the data presented in Table 3. that the higher mango percentage imparted more flavour to mixed fruit toffee. The highest value for flavour was recorded in $\mathrm{C}_{1} \mathrm{~S}_{3}$ (80\% mango $+20 \%$ papaya with $400 \mathrm{~g}$ sugar) while minimum in $\mathrm{C}_{4} \mathrm{~S}_{1}(20 \%$ mango + $80 \%$ papaya with $200 \mathrm{~g}$ sugar) A decreasing pattern of flavour rating value was observed during storage of mixed fruit toffee for 120 days. The reason for this is production of offflavour which adversely affects taste and aroma of product. There are certain enzymatic, physiological or biochemical changes, which result in production of offflavour of product. Similar results were also reported by Punam et al., (2009) who reported that the flavour of bael-mango RTS has higher in initial score but decreased significantly at 90 days of storage. Jakhar and Pathak, (2012) reported that the flavour score decreased continuously during entire period of storage. Similar finding were also reported by Deka et al., (2005).

\section{Taste}

The result obtained about the taste of blended mixed fruit toffee at different stages of storage has been presented in Table 4. It is obvious from the data that the taste of mixed fruit toffee was influenced by the different blend of pulp, sugar ratio and storage period. The score rating decreases continuously with the increase in storage period up to 120 days. However, the best result of mixed fruit toffee for taste were obtained from the treatment combination of $\mathrm{C}_{2} \mathrm{~S}_{3}$ (60\% mango $+40 \%$ papaya pulp with $400 \mathrm{~g}$ sugar) and which was significantly superior in comparison to others. During storage, a significant reduction in taste of mixed fruit toffee was observed. This is due to Production of off-flavour which adversely affects taste of product and there are certain enzymatic, physiological or biochemical changes, which result in production of off-flavour of product. Similar results were found by Punam et al., (2009) who reported that organoleptic quality like taste reduced significantly with increased storage period. These findings are supported by other workers Jakhar and Pathak (2012) in blended RTS of ber and jamun, Deka et al., (2005) in mango-pineapple spiced beverages.

\section{Overall acceptability}

The overall acceptability of mixed fruit toffee is dependent on colour, texture, flavour and taste rating of the product. The results obtained showed that highest score for overall acceptability found in $\mathrm{C}_{2} \mathrm{~S}_{3}$ (60\% mango + $40 \%$ papaya pulp with $400 \mathrm{~g}$ sugar). During storage, it was observed that overall acceptability of mixed fruit toffee was highest at 0 day of storage and it was slightly decreased as the days of storage were increased. Similar results were found by Thakre and Jain (2013) in the blended nectar (50:50) of papaya and banana which was acceptable only for 15 days under ambient condition and up to 45 days under refrigerated condition (Table 5).

The reason for rejection is poor colour, appearance, aroma and taste. Similarly Sakhale et al., (2012) observed that overall acceptability of whey based mango beverage decreased due changes occurred during storage of beverage. Similar results decrease in overall acceptability was found by Singh et al., (2013) with jam prepared from three mango cultivars. Similarly, the findings of Baramanray et al., (1995) showed that the organoleptic rating of freshly prepared product is highly acceptable and reduced significantly with increased storage period. Verma and Gehlot (2006) also reported that overall acceptability decreased during storage period in bael beverages. Similar results were also supported by Jakhar and Pathak (2012) and Manshi et al., (2010) 


\section{Economics}

The data regarding economics of various treatments (recipes) as influenced by various pulp ratio and sugar level has been presented in Table 6. However, clear differences in production cost were recorded with the different treatments and this difference was due to the ratio of pulp and quantity of sugar added with different recipes. In contrast to this the recipes having more proportion of mango pulp and sugar level has recorded more no. of Toffees. Recipes with higher papaya percentage and low sugar level had recorded less number of Toffee. The highest net return of Rs. 271.76 and B:C ratio of 3.11 was obtained in the treatment combination $\mathrm{C}_{2} \mathrm{~S}_{3}$ (60\% mango+ $40 \%$ papaya with $400 \mathrm{~g}$ sugar $/ \mathrm{kg}$ pulp) due to maximum overall acceptability and higher sale rate. While lowest net return of Rs. 62.69 along with benefit cost ratio 1.52 was recorded in treatment combination of $\mathrm{C}_{4} \mathrm{~S}_{1}(20 \%$ mango + $80 \%$ papaya with $200 \mathrm{~g}$ sugar $/ \mathrm{kg}$ pulp). Similarly Parmar (2008) reported that treatment $\mathrm{T}_{3}(75 \%$ guava $+25 \%$ papaya + $62.5 \mathrm{~g}$ sugar $+5 \mathrm{~g}$ citric acid) was regarded as best treatment for mixed fruit leather, Nalage et al., (2014) also reported good B:C ratio for mixed fruit toffee from aonla pulp and ginger extract.

\section{References}

Anonymous. 2014. Indian Horticulture Database-2014, Ed. Saxena M and Gandhi PC. Published by ministry of agriculture, GOI.

Aruna K, Vimala V, Dhanlalakshmi k and Vinodini Reddy. 1999. Physicochemical changes during storage of papaya fruit (Carica papaya L.) bar (Thandra). J. of Fd. Sci. and Techntol. 36 (5): 428-433.

Deka BC, Sethi V and Saikia A. 2005. Changes in quality of mango- pineapple spiced beverage during storage. Indian Journal of Horticulture 62 (1): 65-68.

Hussain I, Sabeen NG, Muhammed RK, Khan TM and Iftikhar S. 2005. Varietal suitability and storage stability of mango squash. Int. J. Agric. Biol. 7:1038-1039.

Jakhar MS and Pathak S. 2012. Studies on the preparation and storage stability of blended Ready-to-serve from ber and jamun pulp. Plant Archives 12 (1): 533-536.

Mamiro P, Fweja L, Chove B, Kinabo J, George V and Mtebe K. 2007. Physical and chemical characteristics of off vine ripened mango (Mangifera indica L.) fruit (Dodo). Afr. J. Biotechnol 6:2477-2483.

Manshi, Gehlot R, Singh R, Siddiqui S. 2010. Development and quality evaluation of jamun (Syzygium cumini L.) cheese and toffee. Haryana Journal of Horticultural Sciences 39 (1/2): 145147.

Matsuura FCAU, Folegatti MIDS, Cardoso RL, Ferreira DC. 2004. Sensory acceptance of mixed nectar of papaya, passion fruit and acerola. Sci. Agricola (Piracicaba, Braz.) 61:604-608.

Nalage AB, Chavan UD and Amarowicz R. 2014. Toffee from aonla and ginger. Italian Journal of Food Science 26 (2): $127-133$.

Parmar H. 2008. Studies on the preparation of guava and papaya mixed fruit leather, its quality and storage. M.sc. Thesis, JNKVV, Jabalpur. 63p.

Punam, Gehlot R, Singh R and Siddiqui S. 2009. Studies on physico-chemical composition of fresh bael and mango fruits. Haryana Journal of Horticultural science 38(1\&2): 66-67.

Sakhale BK, Pawar VN and Ranveer RC. 2012. Studies on the Development and Storage of whey based RTS beverage 
from mango cv. Kesar. J. Food Process Technol. 3 (3): 10144-10148.

Singh J, Sodhi K and Kaur M. 2013. Sensory Evaluation of Jam prepared from Various Cultivars of Mango and Mango- Papaya Blends. Annals of Horticulture 6 (1): 133-138.

Tedjo W, Taiwo K, Eshtiaghi MN and Knorr D. 2002. Comparison of pretreatment methods on water and Comparison of pretreatment methods on water and mangos. J. Food Eng. 53:133-142.

Thakre M and Jain V. 2013. Storage study of blended nectar of papaya and banana under different storage conditions. Progressive Horticulture 45 (1): 100103.

\section{How to cite this article:}

Bharat Kumar, Pandey, C.S. and Rangare, N.R. 2018. Evaluate the Sensory Parameters and Economics of Mixed Fruit Toffee. Int.J.Curr.Microbiol.App.Sci. 7(08): 4321-4333. doi: https://doi.org/10.20546/ijcmas.2018.708.453 